value of $90.2 \%$ for intra-operative detection of diaphragmatic disease

Conclusion* Whilst it is accepted that CT is a poor predictor of diaphragmatic disease, we suggest our figures may be

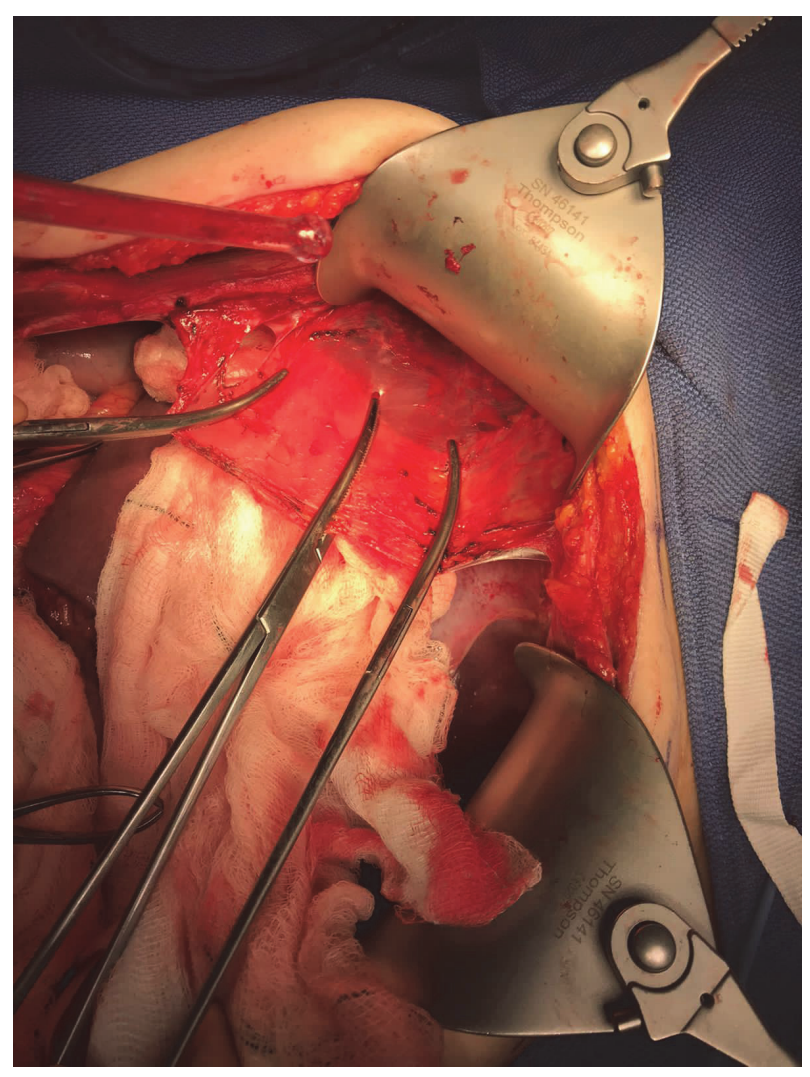

Abstract 543 Figure 1

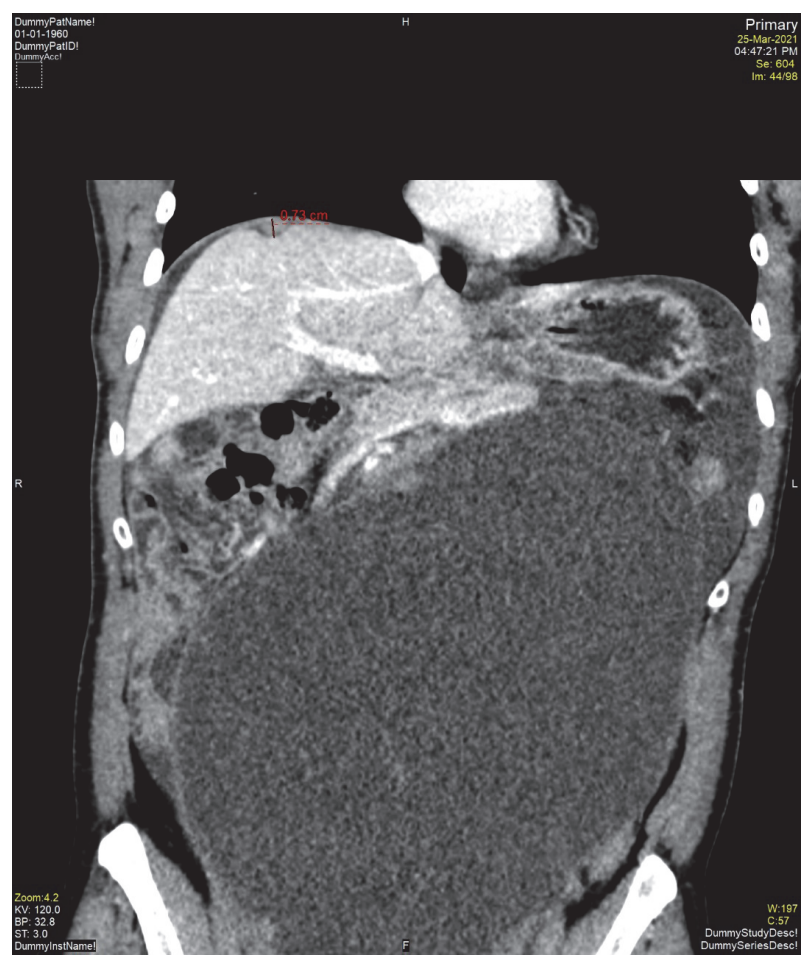

Abstract 543 Figure 2 additionally compounded by a local radiological focus on identification of surgical stopping-points in the context of a unit with a well-established ultra-radical service and experience of diaphragmatic surgery. Gynaecological-oncologists should, however, remain mindful of the limitations of CT and hence approach all relevant cases with the anticipation of encountering diaphragmatic disease.

\section{DIAGNOSTIC AND THERAPEUTIC APPROACHES FOR PATIENTS WITH A STIC LESION - RESULTS OF THE AGO OVAR QUESTIONNAIRE BASED SURVEY IN GERMANY}

1J Van der Ven*, 'V Linz, 'S Krajnak, ${ }^{1} \mathrm{~K}$ Anic, ${ }^{1} \mathrm{R}$ Schwab, ${ }^{2} \mathrm{~S}$ Kommoss, ${ }^{3} \mathrm{~B}$ Schmalfeldt, 4J Sehouli, ${ }^{1} \mathrm{~A}$ Hasenburg, ${ }^{1} \mathrm{M}$ Battista. 'Unimedizin Mainz, Klinik und Poliklinik für Geburtshilfe und Frauengesundheit; '2Universitätsklinikum Tübingen, Department für Frauengesundheit; ${ }^{3}$ Universitätsklinikum Hamburg-Eppendorf, Klinik und Poliklinik für Gynäkologie; ${ }^{4}$ Charité Berlin, Klinik für Gynäkologie mit Zentrum für onkologische Chirurgie

\subsection{6/ijgc-2021-ESG0.425}

Introduction/Background* Despite growing understanding of the carcinogenesis of high-grade serous ovarian cancer and its precursor lesion serous tubal intraepithelial carcinoma (STIC), there is a lack of evidence based recommendations for the clinical management of patients with a STIC lesion.

Methodology We created 23 questions to explore the experience with STIC patients and the diagnostical, surgical and histopathological approaches and used SoSci Survey to host the questionnaire. We invited all German directors of gynecological departments to participate.

Result(s)* 550 colleagues were invited. 131 questionnaires (response rate $24.3 \%$ ) were returned and included in this survey. $45.8 \%$ of the respondents treated one to three STIC patients. $76.0 \%$ of the participants performed opportunistic bilateral salpingectomies during other gynecological interventions. Most of the participants requested the SEE-FIM protocol from their pathologists since 2017. It was used by $54.2 \%$ for prophylactic salpingectomies, by $28.1 \%$ for both prophylactic and opportunistic salpingectomies and by $17.7 \%$ for neither of both. In a case of a STIC lesion 58.8\%, 2.4\%, 37.6\% of participants used the laparoscopic, transvers- or longitudinal laparotomic approach, respectively. The respondents performed a hysterectomy, bilateral ovarectomy or affected side ovarectomy in pre- and postmenopausal patients in $25.6 \%(54.6 \%)$, $24.42(88.4 \%)$ and $50.0 \%(4.6 \%)$, respectively (all p-values $>0.001$ ). Omentectomy, pelvic and para-aortic lymphadenectomy were performed in pre- and postmenopausal women in $60.5 \%$ (63.9\%), 9.30\% (11.6\%) and 9.3\% (11.6\%) (all p-values <0.05).

Conclusion* This survey highlights significant inconsistency in the management of patients with a STIC lesion. Further studies are urgently warranted to elucidate the clinical impact and the necessary therapeutic approach of STIC lesions.

\section{UTERINE SEROUS CARCINOMA MIMICKING ADVANCED OVARIAN CANCER. ARE THERE CLUES TO PRE- OPERATIVE DIAGNOSTIC DIFFERENTIATION? A MINI CASE SERIES}

S Addley*, A Bali, V Asher, S Abdul, A Phillips. University Hospitals of Derby and Burton NHS Foundation Trust, Gynaecology Oncology, UK

10.1136/ijgc-2021-ESG0.426 